\title{
Apnea and Rapid Eye Movement Sleep Excess in the Piglet during Recovery from Hyperthermia
}

\author{
BARBARA C. GALLAND, DAVID P. G. BOLTON, AND BARRY J. TAYLOR \\ Department of Pediatrics and Child Health /B.C.G., B.J.T.J. and Department of Physiolog! /D.P.G.B./. \\ University' of Otago Medical School. Dunedin. New Zcaland
}

\begin{abstract}
This investigation tested the hypothesis that artificially induced mild hyperthermia and recovery from hyperthermia in the developing newborn piglet $(2-10 \mathrm{~d}$ of age) alter sleep state patterns and respiratory control. Continuous measurements of sleep state, respiratory pattern, carbon dioxide production and oxygen consumption were made before, during, and after a 3 -h period of sustained hyperthermia. During hyperthermia, rectal temperatures were raised a mild $1-1.5^{\circ} \mathrm{C}$ above normal, well below the levels likely to cause severe physiologic distress in this species. This resulted in a disruption of the sleep state pattern characterized by a decrease in duration of rapid eye movement (REM) episodes, whereas immediately afterwards, during recovery, the amount of REM sleep increased. In some cases the amount of REM sleep in recovery more than doubled the basal level. Apneas were rarely observed during hyperthermia, but in recovery there was an increase in the total amount of time spent in apnea in both REM and non-rapid eye movement sleep states with a predominance in the REM state. Arousal responses to chemostimulation were not affected at this time. We conclude that the sleeping newborn piglet does indeed show marked changes in sleep state pattern (particularly REM sleep) and in the amount of apnea recorded during and immediately after only a mild increase in core temperature. (Pediatr Res 34: 518-524, 1993)
\end{abstract}

\section{Abbreviations}

$\dot{V}_{2}$, oxygen consumption

$\mathrm{VCO}_{2}$, carbon dioxide production

REM, rapid eye movement

NREM, non-rapid eye movement

TST, total sleep time

SIDS, sudden infant death syndrome

EOG, electrooculogram

The reason for our interest in the relationship between hyperthermia and respiratory stability is the suggestion that SIDS is associated with situations that could give rise to hyperthermia (1-4) or with the postfebrile period (5) rather than hyperthermia itself. The prevalence of REM sleep in these conditions was investigated as a possible cause of respiratory instability, noting that young SIDS victims have been reported as having had a significantly greater number of REM sleep epochs compared with controls (6).

Experimental studies have suggested that mild hyperthermia

Received October 10, 1991; accepted April 22, 1993.

Correspondence: Barbara C. Galland. Ph.D.. Department of Paediatrics and Child Health, P.O. Box 913, Dunedin, New Zealand.

Supported by the Cot Death Division of the National Children's Health Research Foundation of New Zealand. per se could modify respiratory pattern and/or sleep state. In puppies, apnea induced by laryngeal stimulation becomes more severe as the temperature is raised (7), and in premature human infants, raising the environmental temperature enhances the already unstable respiratory pattern characteristic of these infants, i.e. apnea and periodic breathing $(8-10)$. These patterns are particularly enhanced in REM sleep, a state associated with depressed responsiveness to environmental stimuli. In several species, total sleep time increases at the upper limits of thermoneutrality $(11,12)$ and the total duration of REM sleep is correlated with rises in ambient temperature up to this limit (13).

The question this investigation addressed was whether the sleeping newborn showed changes in sleep state and respiratory control during and immediately after artificially induced mild hyperthermia. We chose to conduct our investigations on the young piglet. This species shows postnatal development similar to that of the human infant in terms of sleep state maturation and cardiorespiratory behavior (14), and it is one of the few larger animals born with very limited insulation on the skin surface. The experiments were simple and noninvasive, designed to measure respiration and sleep state during normal sleep, before, during, and after a period of sustained hyperthermia. During this hyperthermia, rectal temperatures were raised 1 to $1.5^{\circ} \mathrm{C}$ above normal, well below the levels likely to cause severe physiologic distress to this species (15).

\section{MATERIALS AND METHODS}

Twenty-four farm-bred piglets of mixed sex and from different litters were separated from the sow $2 \mathrm{~d}$ after birth and thereafter raised on reconstituted milk powder. They were housed singly in a pen where environmental temperature was maintained between 30.5 and $32.0^{\circ} \mathrm{C}$. When possible, all piglets were studied during sleep at 2 and $4 \mathrm{~d}$ after birth (wk 1) and 7 and $10 \mathrm{~d}$ after birth (wk 2). Mean body weights at $2 \mathrm{~d}$ were $1.01 \pm 0.05 \mathrm{~kg}$, and at $10 \mathrm{~d}, 1.43 \pm 0.07 \mathrm{~kg}$.

Sleep Staging. NREM sleep, REM sleep, and the awake state were determined from the signals of standard bipolar EEG $\left(C_{4} /\right.$ $A_{1}$ or $C_{3} / A_{2}$ positions) and EOG (referred to each outer canthus of the eye) surface electrodes. NREM sleep was defined by highvoltage slow waves on the EEG and no eye movements on the EOG. REM sleep was defined by low-voltage waves and eye movements, and wakefulness was defined by low-voltage mixedfrequency EEG pattern with movement artifacts in both the EEG and EOG wave forms. Observations of behavior and respiratory pattern could be used to confirm identification of sleep state. The signals were amplified (Grass 7DA. Grass Instrument Co., Quincy, MA) and relayed through the integrated hardware/ software system of a MacLab (Analog Digital Instruments. Dunedin. New Zealand) for recording and display. TST was calculated as the sum of NREM and REM sleep times within each temperature study phase.

Respiratory Pattern. A respiband (model 150, Respitrace Co., NY) was used to record respiratory pattern from the ribcage and 
abdomen. The output from the Respitrace was relayed through a MacLab system for recording and display. Respiratory pattern was determined from the signal of the sum of the ribcage and abdomen and each trace scanned to identify long expiratory pauses. A pause was defined as an apnea when the expiratory duration was greater than 4 times the average expiratory duration of the previous 10 breaths.

$\dot{V}_{2}$ and $\dot{V} \mathrm{CO}_{2}$. $\dot{\mathrm{VO}}_{2}$ and $\dot{\mathrm{V}} \mathrm{CO}_{2}$ were measured by indirect calorimetry using an adaptation of the method described by Lister et al. (16) for use in neonatal studies. Animals slept in a cylindrical acrylic chamber, $10 \mathrm{~L}$ in volume. Each end was tapered to fit inlet and outlet respiratory tubing. The smaller piglet could turn freely within this chamber and the tubing could be switched depending on which end the animal chose to sleep at. Outside air was sucked through the chamber by means of a vacuum pump (Ameda, Switzerland) at a steady rate of $4 \mathrm{~L} / \mathrm{min}$ as measured with a linear flow meter (rotameter). A large volume chamber was inserted in the line between the flow meter and the pump to damp the pulsatile sucking action of this pump. Concentrations of $\mathrm{O}_{2}$ and $\mathrm{CO}_{2}$ were measured by a mass spectrometer (Perkin-Elmer 1100, Perkin-Elmer Corp., Norwalk, CT). Measurements were continuous, although the single sample line of the mass spectrometer required the inflow and outflow ports to be sampled alternately. A MacLab system was used to record and display the signals. The measured outflow for $\mathrm{O}_{2}$ ranged between 20.5 and $19.9 \%$, and for $\mathrm{CO}_{2}$, between 0.4 and $0.9 \%$. The lowest and highest values for $\mathrm{O}_{2}$ and $\mathrm{CO}_{2}$, respectively, were recorded during hyperthermia. $\dot{\mathrm{V}}_{2}$ and $\dot{\mathrm{V}}_{\mathrm{CO}_{2}}$ were calculated as the product of the differences between inflow and outflow gas concentrations and flow rate, and corrected for standard temperature and pressures dry. The Haldane correction factor (17) was used in the calculation of $\dot{\mathrm{V}}_{2}$. The system was calibrated for $\dot{\mathrm{V}}_{2}$ and $\dot{\mathrm{V}}_{\mathrm{CO}_{2}}$ by injecting $\mathrm{N}_{2}(150-200 \mathrm{~mL} / \mathrm{min})$ and $\mathrm{CO}_{2}(8-$ $12 \mathrm{~mL} / \mathrm{min})$, respectively, while air was sucked through at different flow rates $(1-7 \mathrm{~L} / \mathrm{min})$. The flow rate of $4 \mathrm{~L} / \mathrm{min}$ was found to be sufficient to trap all outflow gases without causing too much dilution with the inflow air. The average errors between the measured and theoretic values for $\dot{\mathrm{V}}_{2}$ and $\dot{\mathrm{V}}_{2}$ at this air flow rate were \pm 1.89 and $3.88 \%$, respectively. Respiratory exchange ratios were calculated from the division of $\dot{\mathrm{V}}_{2}$ by $\dot{\mathrm{V}}_{2}$.

Temperature Recording. Thermistors and a multichannel data logger (Squirrel 1200) were used to measure and record temperatures every $30 \mathrm{~s}$ from ambient and room air and from the forehead, ear, trunk, and rectum. The same system recorded relative humidity within the chamber by a capacitive humidity probe. Humidity fluctuated with changes in temperature but generally was maintained between 50 and $70 \%$ throughout experiments.

Hypoxic Challenge. Hypoxic challenges were performed in some of the heated experiments. For this, the suction rate of gases throughout the chamber remained steady at $4 \mathrm{~L} / \mathrm{min}$. First, the air inlet valve was switched to a Douglas bag containing $\mathrm{N}_{2}$ mixed with $3 \% \mathrm{CO}_{2}$. Once the $\mathrm{O}_{2}$ content of the air in the chamber had dropped to approximately $14 \%$, the valve was switched open to another bag containing the final hypoxic gas level of $10 \% \mathrm{O}_{2}$ mixed with $3 \% \mathrm{CO}_{2}$ to maintain normocapnia. These gas levels were reached within $90 \mathrm{~s}$ of first replacing the air with $\mathrm{N}_{2}$. Each test involved the animals breathing $10 \% \mathrm{O}_{2}$ mixed with $3 \% \mathrm{CO}_{2}$ until arousal occurred or, if arousal did not occur, for a maximum of $300 \mathrm{~s}$ at which time the stimulus was withdrawn. Hypoxic challenges in NREM sleep were always complete for that sleep state, but sometimes in REM sleep animals failed to arouse before a change to the NREM state occurred. In these instances, the arousal latency was still assigned to REM sleep because the hypoxic test was initiated in this state. Latency to arousal was measured from the time when the gas mixture reached the desired level within the chamber to the time when waking was evident from EEG recordings or movement.

Study design. Eight piglets (heated group) were studied on four occasions during which measurements were made in normothermic conditions, in hyperthermic conditions after being warmed, and during cooling after hyperthermia (recovery). The studies were performed at 2 and $4 \mathrm{~d}$ of age (wk 1) and at 7 and $10 \mathrm{~d}$ of age (wk 2). During wk 2, sedation was necessary (chloral hydrate; $160 \mathrm{mg} / \mathrm{kg}$ dissolved in the milk feed) to be sure of sufficient sleep time for the study.

To evaluate the effects of sedation, another eight piglets (sedation control group) were investigated on $d 4$ according to the same protocol as the heated group (see below) except for sedation with chloral hydrate $(160 \mathrm{mg} / \mathrm{kg})$. The results were compared with those recorded in the heated group on $\mathrm{d} 4$ without sedation.

To evaluate any time-dependent changes independent of warming, another eight animals (unheated group) were investigated over a 6 -h period on $\mathrm{d} 2,4,7$, and 10 as for the heated group, but they were kept in thermoneutral ambience. Here also sedation was used during the wk-2 studies only. All the data presented for the heated group are compared with data from these animals.

An additional three animals (blood gas group) were chronically catheterized to allow blood gas analysis during the protocol for the heated group as described below. They were operated on $2 \mathrm{~d}$ before study with full sterile procedures under general anesthesia ( $1.5 \%$ halothane in oxygen); a catheter was tied into a carotid artery and brought subcutaneously to emerge at the back of the neck. They were dosed regularly with antibiotics and the catheters flushed with heparinized saline $(10 \mathrm{U} / \mathrm{mL})$. All three animals were studied on $\mathrm{d} 4$, two on $\mathrm{d} 7$, and one on $\mathrm{d} 10$ and were sedated as above. Blood samples $(0.3 \mathrm{~mL})$ were taken hourly and analyzed using an ABL330 Acid-Base Laboratory (Radiometer. Copenhagen, Denmark).

Study Protocol. Heated group. Thirty min after a scheduled feed, the animal was prepared for study and placed in the prewarmed acrylic chamber. This was housed inside a convective incubator (Air-Shields, Air-Shield Inc., Hatboro, PA), allowing its temperature to be controlled. This was initially in the thermoneutral range as determined from preliminary studies: $31-$ $33^{\circ} \mathrm{C}$ for 2 - and 4 -d-old piglets, $29-31^{\circ} \mathrm{C}$ for 7 and 10 -d-old piglets. The baselıne records were obtained durıng $5 U$ to $6 U \mathrm{~m} ı n$ at this setting and during the period of increased chamber temperature causing a slow risc in rectal temperature lasting 1.5 to $2 \mathrm{~h}$ in all. The hyperthermia records were obtained over a 3-h period during which the rectal temperature was maintained between 40.0 and $40.5^{\circ} \mathrm{C}$. Recovery data were recorded over the period of approximately $1 \mathrm{~h}$ of cooling back to the original rectal temperature and 30 to $60 \mathrm{~min}$ of maintenance at that temperature.

Unheated group. Ambient temperature was maintained to keep animals within their thermoneutral range for the entire study period (approximately $6 \mathrm{~h}$ ). The data were divided into periods of 120,180 , and 120 min to match the mean times for baseline, hyperthermia, and recovery in the time sequence of the heated group.

All studies were performed over the same period of the day beginning at $0900 \mathrm{~h}$. Animals slept through the study period in the dark, and as long as they were undisturbed, they slept readily and with seemingly little discomfort. This study protocol was approved by the Committee on Ethics in the Care and Use of Laboratory Animals of the University of Otago, Dunedin. New Zealand.

Data Collection. Respiratory pattern, temperatures, EEG, and EOG were recorded continuously. Hypoxic challenges were performed in several of the animals in the temperature study group. Challenges were performed early in the baseline period and at the end of the recovery phase in both REM and NREM sleep states. Data recorded for $\dot{\mathrm{V}}_{2}$ and $\dot{\mathrm{V}}_{\mathrm{CO}}$ measurement were interrupted when this challenge took place and for approximately 15-20 min thereafter. To ensure steady state for each change in sleep state, $\dot{\mathrm{VO}}_{2}$ and $\dot{\mathrm{V}}_{\mathrm{CO}_{2}}$ data were discarded for the first minute of recording and thereafter averaged over the sleep state and pooled for each thermal phase.

Statistical Analysis. Unless otherwise stated, data are presented as the mean \pm SEM. Nonparametric analysis using the Wilcoxon 
sign-ranked test was used to compare I) differences within each study group for thermal condition and age; 2) time-dependent changes independent of heating by comparing the heated group data with the unheated group data; and 3) the effects of sedation by comparing the sedation control group data with the data from the heated group of animals studied on $\mathrm{d} 4$ without sedation. A sign test was used to compare the ability of animals to arouse during hypoxic gas stimulation. The conditions for the sign test were 1 ) arousal occurring within the period of hypoxic stimulation ( $300 \mathrm{~s})$ and, 2) failure to arouse within this time.

Initial analyses of the data to observe differences in the various physiologic parameters recorded within the first and second weeks of study (d 2 and 4 and 7 and 10, respectively) showed no significant changes or consistent trend. The data were then broken into the two age groups (wk 1 and wk 2) for final comparisons and are presented in this way. $p$ values for all data $<0.05$ were accepted as the level of statistical significance.

\section{RESULTS}

$\dot{V}_{\mathrm{O}_{2}}, \dot{V}_{\mathrm{CO}}$, and respiratory rate. The mean values for $\dot{\mathrm{V}}_{\mathrm{O}_{2}}$, $\dot{\mathrm{V}} \mathrm{CO}_{2}$, and respiratory rate associated with changes in thermal condition are given for the heated group of animals in Table 1. Data were averaged from the continuous records obtained during NREM sleep. Records of these data in REM sleep were often variable because of gross body movements and were thus not included in the analysis. Generally, $\mathrm{V}_{2}$ increased when rectal temperature rose above $40.0^{\circ} \mathrm{C}$ (hyperthermia); this increase was not statistically significant but nevertheless represented a period of slightly raised metabolic activity. If ambient temperature went low enough to allow a fall in rectal temperature $\left(<39.0^{\circ} \mathrm{C}\right), \dot{\mathrm{V}}_{2}$ began to rise. Measurements of $\mathrm{V}_{2}$ were lowest in baseline and recovery phases and were therefore considered to be in the range of thermoneutrality. For the unheated group, values for $\dot{\mathrm{V}}_{2}$, $\mathrm{VCO}_{2}$ did not change over time and were similar to the values recorded in the heated group at thermoneutrality. Respiratory exchange ratios remained within normal limits $(0.80-0.85)$ during all phases of each experiment.

Respiratory pattern during hyperthermia was characterized at all ages by a rapid respiratory rate and a small tidal volume (as determined from the breath amplitude) typical of a panting species showing no change as a result of repeated heat exposure. Respiratory rate and tidal volume gradually returned to baseline values during recovery.

Sleep state. The amount of time in each sleep state and the duration of episodes were calculated within each study phase. Any periods of hypoxic challenge were excluded. REM duration was generally not long (approximately 2 to $4 \mathrm{~min}$ ), and if a REM episode was in progress during a change in thermal phase, that episode was assigned to the phase in which it was initiated. Figure 1 shows the relative amounts of time spent in REM sleep expressed as a percentage of the TST. TST for the heated group during baseline, hyperthermia, and recovery phases were 106.5 $\pm 6.6,147.2 \pm 9.3$, and $99.1 \pm 7.9 \mathrm{~min}$, respectively, during $w \mathrm{k}$ $1 ;$ in wk 2 they were $108.6 \pm 11.8,135.1 \pm 7.1$, and $95.5 \pm 9.0$

Table 1. Mean values $( \pm S E M)$ for $\dot{V} \mathrm{O}_{2}, \dot{V} \mathrm{CO}_{2}(\mathrm{~mL} / \mathrm{kg} \cdot \mathrm{min})$, and respiratory frequency $(f)$ obtained from heated group of animals*

\begin{tabular}{lrcr} 
& \multicolumn{1}{c}{$\mathrm{f}$} & \multicolumn{1}{c}{$\dot{\mathrm{V}} \mathrm{O}_{2}$} & \multicolumn{1}{c}{$\dot{\mathrm{V}} \mathrm{CO}_{2}$} \\
\hline Wk $1(n=16)$ & & & \\
Baseline & $35.5 \pm 14.2$ & $14.0 \pm 0.6$ & $10.6 \pm 0.4$ \\
Hyperthermia & $234.1 \pm 11.8$ & $15.2 \pm 0.6$ & $11.9 \pm 0.4$ \\
Recovery & $33.4 \pm 18.2$ & $14.1 \pm 0.8$ & $10.8 \pm 2.2$ \\
Wk 2 $(n=14)$ & & & \\
Baseline & $29.8 \pm 14.3$ & $12.2 \pm 0.5$ & $9.5 \pm 0.4$ \\
Hyperthermia & $255.3 \pm 17.7$ & $13.8 \pm 0.7$ & $10.6 \pm 0.5$ \\
Recovery & $33.9 \pm 11.5$ & $12.8 \pm 0.5$ & $9.7 \pm 0.3$ \\
\hline
\end{tabular}

* Data are separated by age at study and thermal condition.

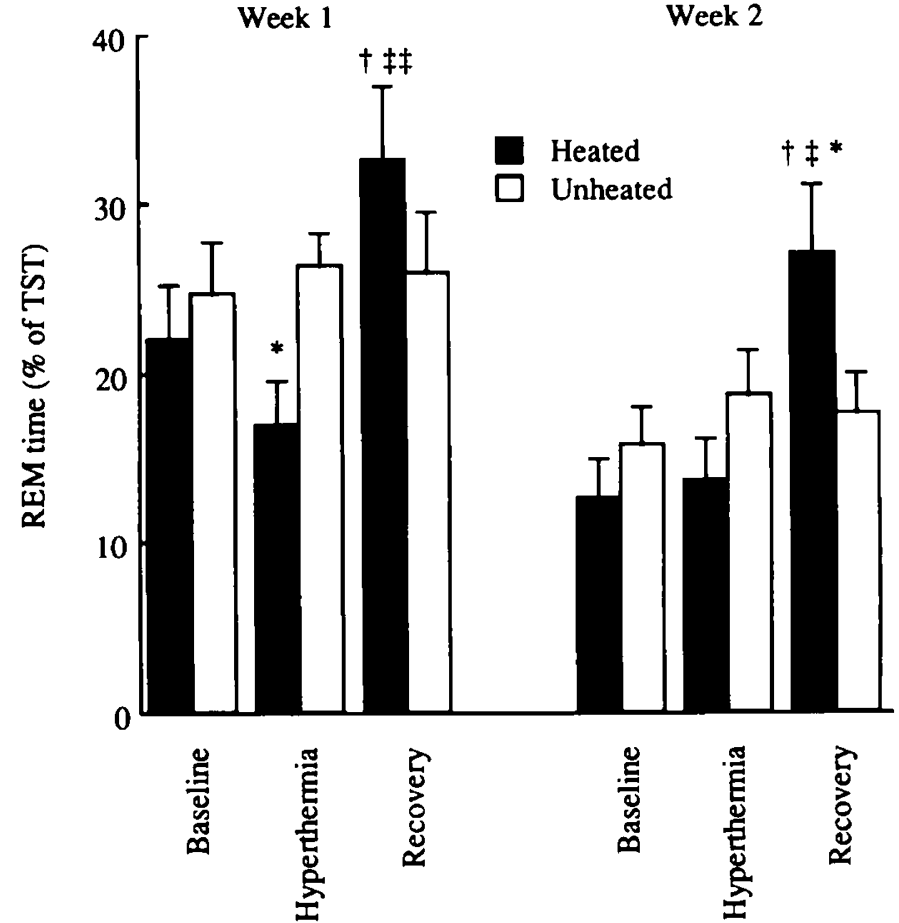

Fig. 1. Mean values ( \pm SEM) of the relative amount of time spent in REM sleep expressed as a percentage of TST. Data are given for wk 1 and 2. For the heated group $(n=16)$, the values represent data from baseline, hyperthermic, and recovery time periods, and for the unheated group ( $n=16$ ), the values represent data matched for these same time periods recorded during conditions of thermoneutral ambience. $\uparrow, p<$ 0.05 v's baseline; $\ddagger . p<0.05$ and $\ddagger \neq, p<0.001$ vs hyperthermia; ${ }^{*}, p<$ 0.05 vs unheated.

min. TST in the unheated group was similar only during the hyperthermic time phase of wk 2 . TST was significantly $(p<$ $0.05)$ longer $(150.9 \pm 6.2 \mathrm{~min})$ than the corresponding hyperthermic phase for the heated group.

Age comparisons of the amount of REM sleep during the baseline phase in the heated and unheated group (all three phases) showed a general decrease in wk 2 compared with wk 1 , although these differences were not statistically significant (Fig. 1). When data were compared between each thermal phase for the heated group, the amount of time spent in REM sleep during recovery was significantly greater than during baseline and hyperthermic phases. In several individual cases, the percentage of REM sleep in recovery more than doubled the basal level. In wk 2, the greater amount of REM sleep resulted from an increase in the duration of REM episodes (Fig. 2). In the hyperthermic phase of the heated group, REM sleep duration was significantly shorter than baseline and recovery, probably because the animals awoke more often and were generally more unsettled at this time. These differences noted in the amount and duration of REM sleep in the heated group of animals with changing thermal phases were indeed independent of time because statistical significance of most of these differences still held when data were compared with the unheated group of animals studied over the same time periods at thermoneutral ambience (Figs. 1 and 2 ).

Apneas. The total length of apneic pauses was calculated within NREM and REM sleep states and the amount of apnea expressed as a percentage of time spent in each state. This was calculated for only baseline and recovery phases because apneas were rarely observed during hyperthermia. In both heated (baseline phase) and unheated animals (baseline and recovery phases) during wk 1 , REM sleep contained significantly more $(p<0.05)$ apnea than NREM sleep (Fig. 3); however, this was not evident during wk 2 . In the heated group, there was a marked increase in the amount of apnea during recovery compared with the baseline 


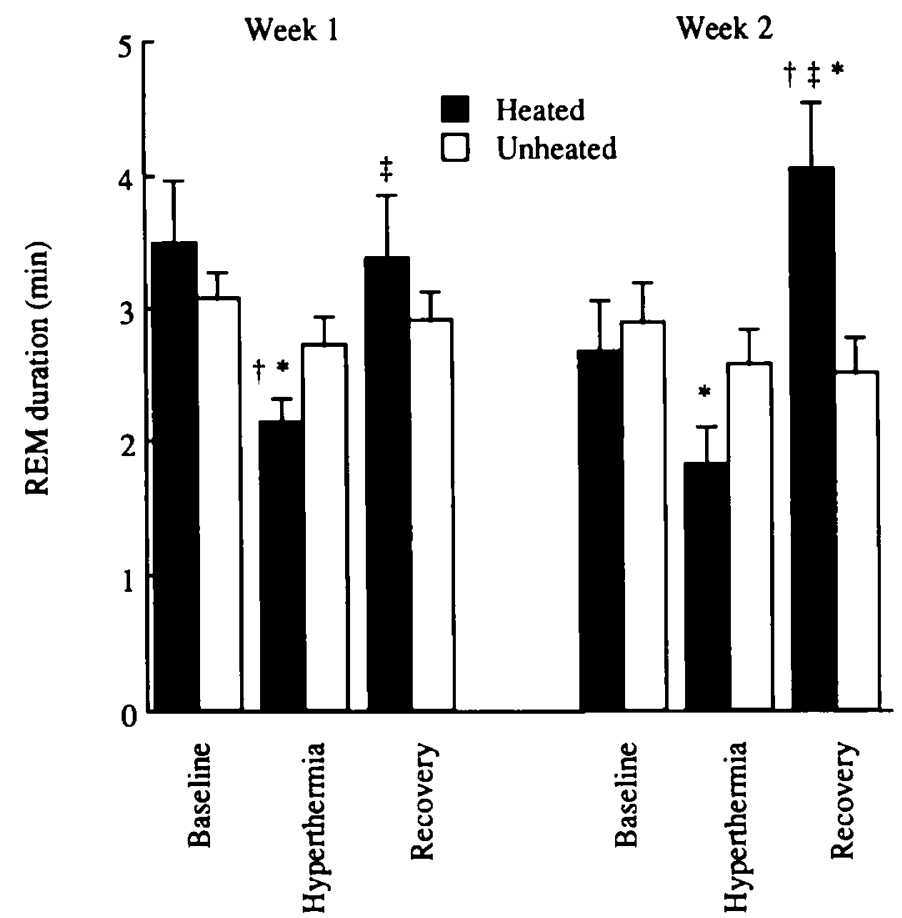

Fig. 2. Mean values ( \pm SEM) of the duration of REM episodes occurring in wk 1 and wk 2. For the heated group $(n=16)$, the values represent data from baseline, hyperthermic, and recovery time periods, and for the unheated group $(n=16)$, the values represent data matched for these same time periods recorded during conditions of thermoneutral ambience. $\dagger, p<0.05$ is baseline; $\ddagger . p<0.05$ is hyperthermia: ${ }^{*}, p<$ 0.05 is unheated.

phase. This increase occurred not only in REM sleep, but also in the NREM state, and was evident at each age of study. The increase in the amount of apnea could be truly attributed to the thermal phase of recovery and not to an effect of time, because the number of apneas occurring in the corresponding time period for "recovery" in the unheated group was much less and did not differ from baseline. The absolute length of apnea depended on the respiratory rate of the animal at the time, but generally the range was between 1 and $6 \mathrm{~s}$, the longest pauses often following a sigh.

Effects of sedative on sleep state and apnea. For the heated protocol used on animals studied on $\mathrm{d} 4$, sedation slightly depressed the amount of REM sleep, but this was significant only during the baseline phase, not during hyperthermia or recovery phases (Table 2). Sedation had no significant effect on REM duration but did decrease the amount of time spent awake. Sedation appeared to have no effect on the amount of apnea recorded. For sedated animals, the percentage of time spent in apnea during baseline and hyperthermic phases for NREM sleep was $1.2 \pm 0.5$ and $2.3 \pm 0.7$, respectively; for REM sleep, it was $0.9 \pm 0.6$ and $2.7 \pm 0.5$. In nonsedated animals, these percentages were: NREM, $0.3 \pm 0.1$ and $1.2 \pm 0.7$; and REM, $1.6 \pm 0.5$ and $4.0 \pm 0.8$.

Arousal to hypoxic challenge. Six of the eight animals from the heated group were challenged with hypoxic gas mixtures. The cumulative percentages of animals failing to arouse were calculated over the $300 \mathrm{~s}$ of hypoxic stimulation (Table 3). No significant differences were apparent when data were compared between baseline and hyperthermic phases in NREM or REM sleep. Furthermore, data to compare arousal failures between the two sleep states showed no significant difference.

Arterial PCO. To determine whether the amount of apnea during recovery could be attributed to a lowered arterial $\mathrm{CO}_{2}$ level, a separate series of four experiments was performed. Blood samples were collected from animals that were subjected to temperature variations as in the heated group protocol. The data for each animal are given in Figure 4. Arterial $\mathrm{PCO}_{2}$ fell approximately $10 \mathrm{~mm} \mathrm{Hg}(1.3 \mathrm{kPa})$ toward the end of the hyperthermic period when respiratory frequency was high and showed a slight but incomplete return to baseline during the recovery phase.

\section{DISCUSSION}

The most interesting findings from these studies were that during recovery from hyperthermia all piglets showed a marked increase in two variables: 1 ) the amount of time spent in REM sleep, and 2) the total amount of apnea in both REM and NREM sleep. There is nothing in the protocol that could distort the sleep patterns, with the possible exception of the chloral hydrate
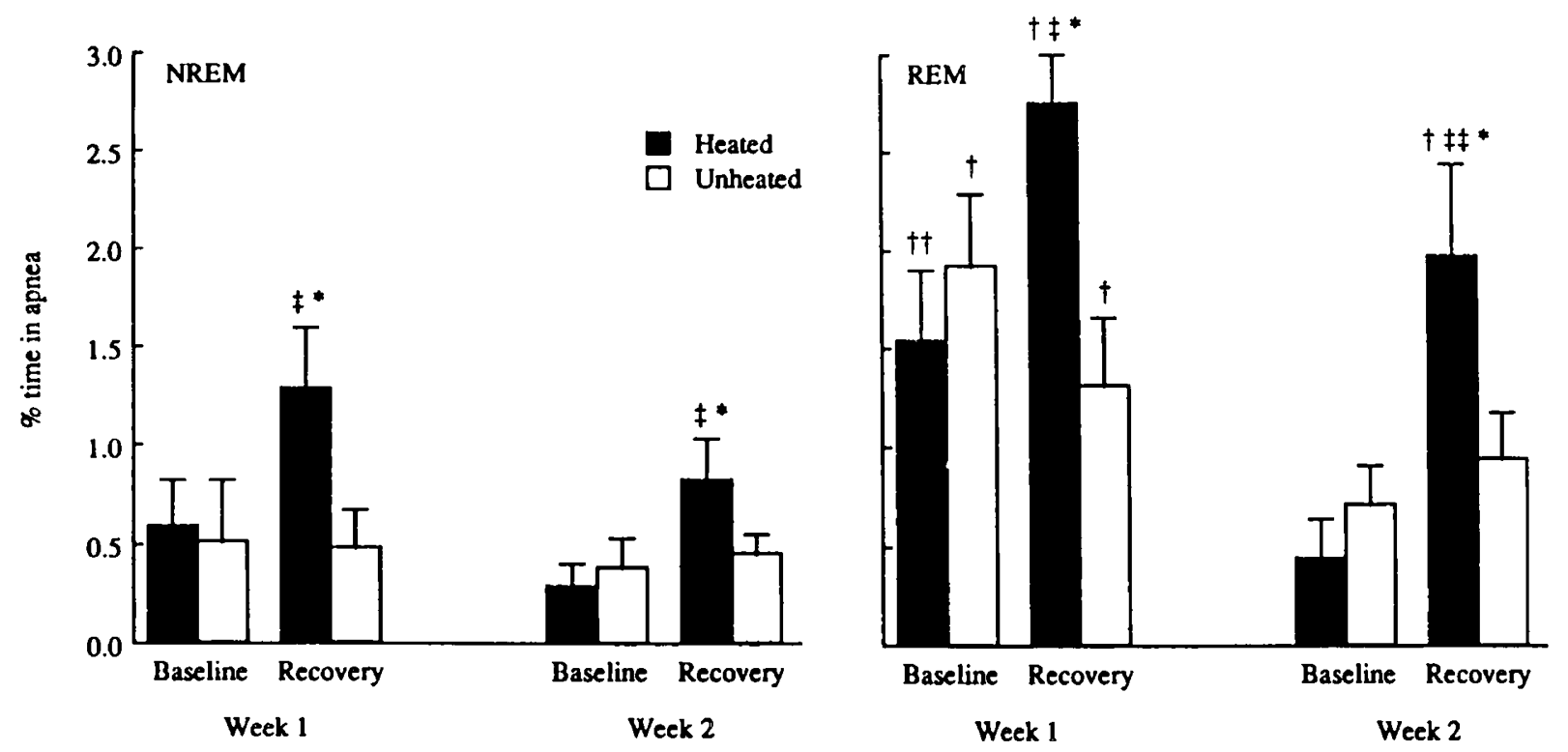

Fig. 3. Mean values ( \pm SEM) of the amount of time spent in apnea expressed as a percentage of NREM (left graph) and REM (right graph) sleep states occurring in wk 1 and 2. For the heated group $(n=16)$, the values represent data from baseline and recovery time periods, and for the unheated group $(n=16)$, the values represent data matched for these same time periods recorded during conditions of thermoneutral ambience. $\dagger$, $p<0.05$ is NREM: $¥ . p<0.05$ is baseline: ${ }^{*}, p<0.05$ is unheated. 
Table 2. Comparison of sleep state parameters between sedated piglets and sedation control group of piglets (nonsedated) studied 4 $d$ after birth*

\begin{tabular}{|c|c|c|c|c|c|c|c|c|c|}
\hline & \multicolumn{3}{|c|}{$\begin{array}{l}\text { Amount of REM } \\
(\% \text { of TST })\end{array}$} & \multicolumn{3}{|c|}{$\begin{array}{l}\text { Average REM duration } \\
\text { (min) }\end{array}$} & \multicolumn{3}{|c|}{$\begin{array}{c}\text { Awake } \\
(\% \text { of TRT) }\end{array}$} \\
\hline & Baseline & Hyperthermia & Recovery & Baseline & Hyperthermia & Recovery & Baseline & Hyperthermia & Recovery \\
\hline $\begin{array}{l}\text { Sedated animals } \\
\quad(n=8)\end{array}$ & $17.7 \pm 3.7+$ & $11.8 \pm 1.5$ & $27.3 \pm 5.2$ & $2.5 \pm 0.5$ & $2.2 \pm 0.3$ & $3.0 \pm 0.4$ & $7.9 \pm 2.0 \dagger$ & $10.7 \pm 2.1 \dagger$ & $11.2 \pm 3.1$ \\
\hline $\begin{array}{l}\text { Nonsedated ani- } \\
\text { mals }(n=8)\end{array}$ & $24.3 \pm 3.3$ & $14.4 \pm 2.1$ & $32.2 \pm 6.7$ & $3.7 \pm 0.4$ & $1.88 \pm 0.5$ & $3.6 \pm 0.2$ & $12.3 \pm 2.8$ & $16.2 \pm 1.2$ & $14.6 \pm 6.2$ \\
\hline
\end{tabular}

* TRT, total recording time.

$+p<0.05$ vs nonsedated.

Table 3. Percentage of animals arousing within period of hypoxic gas stimulation $(300 \mathrm{~s})^{*}$

\begin{tabular}{cccccc}
\hline & \multicolumn{2}{c}{ Baseline $(\%)$} & & \multicolumn{2}{c}{ Recovery (\%) } \\
\cline { 2 - 3 } \cline { 5 - 6 } WkEM sleep & Wk 1 & Wk 2 & & Wk 1 & Wk 2 \\
\hline \multirow{2}{*}{ REM sleep } & $(n=12)$ & $(n=12)$ & & $(n=10)$ & $(n=8)$ \\
& 83.3 & 87.5 & & 33.3 & 63.6 \\
& $(n=12)$ & $(n=9)$ & & $(n=10)$ & $(n=7)$ \\
& 50.0 & 44.4 & & 20.0 & 80.0 \\
\hline
\end{tabular}

* Data are separated by age at study, temperature condition, and sleep state.
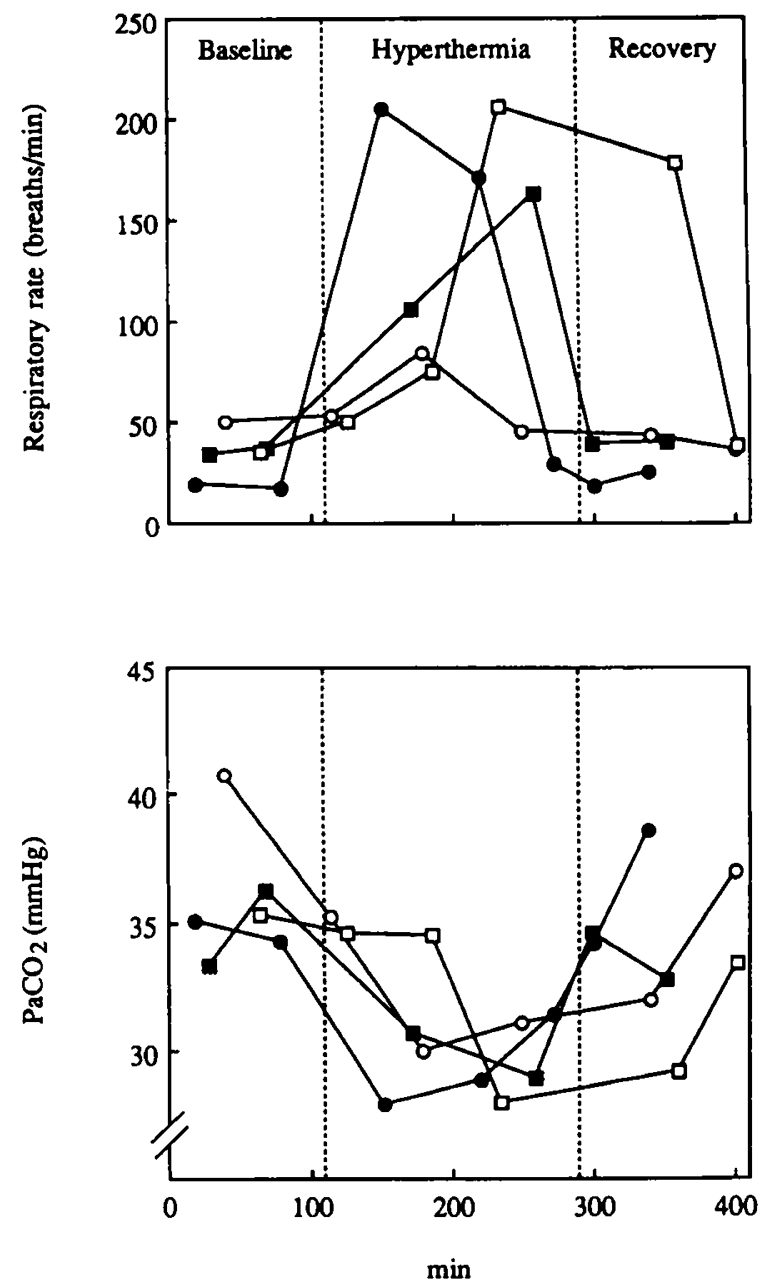

Fig. 4. Respiratory rate (breaths/ $\mathrm{min}$ ) and corresponding arterial levels of $\mathrm{CO}_{2}\left(\mathrm{PaCO}_{2}\right)$ recorded from piglets subjected to the heated protocol and aged $4(\Theta), 4(O), 7(\square)$, and $10(\square) \mathrm{d}$. SI conversion: $1 \mathrm{~mm} \mathrm{Hg}=$ $0.1333 \mathrm{kPa}$. sedation in the older animals. Measurements were noninvasive and the ambient temperature was never in the range to produce discomfort or shivering. Chloral hydrate was chosen as the sedative because of its reported lack of effect on REM sleep state $(18,19)$ and ventilatory responses to hypoxia or hypercapnia (19. 20). Our data suggest that chloral hydrate had a slight depressive effect on the amount of REM sleep but not REM duration. Sedation may therefore have influenced the apparent decrease in the proportion of REM sleep in wk 2 compared with wk 1, although our data would appear to be consistent with a "maturation" of sleep staging as observed by Kuipers and Whatson (21) in nonsedated piglets up to 5 wk of age. Because sedation did not influence the amount of REM sleep in the other two thermal phases, we feel that it was unlikely to have a significant effect on the observed changes in sleep state with temperature. Not surprisingly, use of the sedative decreased the amount of time spent awake, a trend that followed through all thermal phases. Sedation did not, however, influence the amount of apnea recorded, the observed decrease with age probably representing a maturing respiratory control system.

Hyperthermia altered the sleep pattern of the piglet during both weeks of study. The duration of individual REM episodes was reduced, and the total amount of REM sleep was depressed. The animals were more unsettled and REM periods were often interrupted by waking soon after onset of REM. Several studies have monitored sleep behavior in the adult human exposed to acute episodes of hyperthermia (22-24). Most report a disruption of the sleep pattern also, in particular the frequency and duration of REM episodes. Raised core temperature constitutes a thermal load, which may be accentuated by a fall in the hypothalamic set point temperature occurring at the onset of sleep $(25,26)$. If this coincides with an ambient temperature high enough to frustrate heat-losing behavior, then thermal stress may be said to occur. Simultaneous core and peripheral thermal receptor activation can be one form of stress disrupting sleep patterns, whether or not some adaptation to high ambient temperature can be observed. Adult men, adapted to thermoregulate satisfactorily in hot environments, still showed a disturbed sleep pattern when subjected to heat exposure (27).

In the present study, recovery from hyperthermia resulted in a marked increase in the amount and duration of REM sleep in the piglet. An increase in REM sleep can occur as a rebound after REM deprivation in hyperthermia. Studies in adult cats suggest that a daily quota exists of one of the EEG characteristics of REM sleep, the pontine-geniculate-occipital spikes, and that any deprivation of pontine-genticulate-occipital spikes during one period results in a catch-up later on (28). Cats exposed to cold conditions showed a rebound increase in REM sleep only when the mean total duration of the REM epochs fell below $30 \%$ of the original value at thermal neutrality (29). In our studies, a small attenuation of REM sleep ( $16 \%$ ) during hyperthermia was evident only in the first week of study, although REM sleep was enhanced in both weeks during recovery (Fig. 2). Perhaps the differences are that, because REM sleep is more common in the newborn than in the more mature animal, only 
small debts in REM sleep need occur to induce a postdeprivation rebound.

An alternative explanation for the observed sleep state changes during recovery is that removal of the stress associated with the hyperthermic stimulus (high core temperature and high ambient temperature) induced REM sleep in these piglets. Sleep studies in the hyperthermic adult rat have shown that even brief periods of falls in ambient air temperature toward thermoneutrality resulting in skin cooling can induce REM sleep (30). In the piglet, ambient temperature fell quite quickly as the thermal stress was removed; the skin temperature responded abruptly to this change, whereas rectal temperature showed a considerable delay. This explanation would account for the increase in REM sleep observed in both wk 1 and 2, inasmuch as the study protocols were identical.

Apneas were more common in the piglet in REM sleep during baseline recordings, but the frequency decreased as the temperature was raised toward the upper limits of thermoneutrality and consequently apneas were rarely observed during the period of sustained hyperthermia. These findings differ from those of the premature human infant in which apneas were more frequent at the upper end of the thermoneutral zone $(8,10)$ and the duration of periodic breathing during REM sleep was increased as the temperature was raised, but still within the limits of thermoneutrality (9)

Under hyperthermic conditions, the relative absence of apneas in the piglet was not unexpected because this species responds to thermal stress by increasing ventilation in the form of highfrequency, low-tidal-volume ventilation, i.e. panting. This thermal drive to ventilation would probably overcome any other central or peripheral inhibitory influences on breathing. Even during the limited amount of REM sleep when the fast respiratory rates of panting were mildly attenuated, apneas were never observed. Although the baseline rates of respiration are not dissimilar between the newborn pig and human infant, infants exposed to heat cannot increase their respiration anything like the 3- to 4-fold increase in rate observed in the piglet. The ways in which heat is lost by evaporation account for these different responses. In the infant, this is mainly by the process of sweating (31), whereas in the piglet, by far the major evaporative heat loss is achieved by the increase in respiratory rate (32). Sweat glands do exist in the piglet (33), but these are mainly inactive.

During recovery from hyperthermia there was an increase in the total amount of time spent in apnea during both REM and NREM sleep, again with a predominance in the REM state. The absence of thoracic or abdominal movements during the pauses would suggest that the apneas were central in origin. Results from the four experiments in which we were able to analyze blood gases showed that mild hypocapnia resulted from the panting of hyperthermia, and this persisted through the recovery period. Adult humans do not show the same ability to pant without increasing alveolar ventilation ("primary panting"), and when heated to this degree tend to become so hypocapnic that they exhibit the cardopedal spasms of tetany $(34,35)$. If, as seems likely, some of the apnea could be attributed to the fall in arterial $\mathrm{PCO}_{2}$ to $30 \mathrm{~mm} \mathrm{Hg} \mathrm{(4} \mathrm{kPa)} \mathrm{in} \mathrm{the} \mathrm{piglets,} \mathrm{then} \mathrm{the} \mathrm{human} \mathrm{baby}$ could be much more affected in this way. We do not know of any experiments on human babies to characterize their response to hyperthermia, nor of an ethical committee that would approve such an investigation. Alternatively, the increase in apneas in recovery could be attributed to the effects of sleep deprivation in hyperthermia as reported in infants after short-term sleep deprivations (36).

Our findings that REM sleep and apnea in REM and NREM sleep are increased during recovery from hyperthermia suggest that there could be an overall increased risk of failure to arouse from respiratory stressors. During REM sleep, arousal is depressed in dogs (37), lambs (38), and calves (39). In contrast, the adult human shows no sleep state difference in arousal from hypoxemia (40), although for obvious reasons very few studies of this nature have been performed. We did not observe any difference in failure to arouse from hypoxic challenges between baseline and recovery phases (Table 3 ). Neither could we detect any difference between sleep states. Responses were, however, extremely variable and showed no consistent pattern. Many could have woken independent of the stimulus, especially because it took $90 \mathrm{~s}$ to complete the change before timing of arousal states. REM sleep may be associated with poor arousal from various stimuli including hypoxia, but (in our studies) apneic breathing during the recovery phase is not dependent on the prevalence of REM. The possibility still exists that arousal responses to experimental stimuli that produce rapidly developing hypoxemia, such as airway obstruction or rebreathing, could be impaired during this recovery phase. In studies on newborn piglets of an age similar to those used in this study, arousal after airways obstruction in REM sleep was significantly delayed compared with NREM sleep (41).

In summary, our noninvasive study of the newborn piglet showed that during recovery from hyperthermia there was an increase in the amount of apnea in both REM and NREM sleep states and an increase in the amount of REM sleep per se. An increase in the amount of apnea and REM sleep has been associated with some cases of $\operatorname{SIDS}(6,42)$. Our data were recorded during and after short-term exposure to exogenous hyperthermia $(3 \mathrm{~h})$. Further research is required to test the effects of recovery from long-term exposure to hyperthermia or fever.

Acknowledgment. The authors thank Sheila Williams for her statistical advice.

\section{REFERENCES}

1. Stanton AN 1984 Overheating and cot death. Lancet 2:1199-120

2. Nelson EAS, Taylor BJ, Weatherall IL 1989 Sleeping position and infant bedding may predispose to hyperthermia and sudden infant death syndrome. Lancet $\mathrm{I}: 199-201$

3. Fleming PJ, Gilbert R, Azaz Y. Berry PJ, Rudd PT. Stewart A. Hall E 1990 Interaction between bedding and sleeping position in the sudden infant death syndrome: a population based case-control study. Br Med J 301:85-89

4. Ponsonby A-L, Dwyer T, Gibbons LE. Cochrane JA. Jones ME. McCall MJ 1992 Thermal environment and sudden infant death syndrome: case-control study. Br Med J 304:277-282

5. Nelson EAS, Taylor BJ, Mackay SC 1989 Child care practices and the sudden infant death syndrome. Aust Paediatr J 25:202-204

6. Schechtman VL. Harper RM. Wilson AJ. Southall DP 1992 Sleep state organization in normal infants and victims of the sudden infant death syndrome. Pediatrics 89:865-870

7. Haraguchi S. Fung RQ. Sasaki CT 1983 Effect of hyperthermia on the laryngeal closure reflex: implications in sudden infant death syndrome. Ann Otol Rhinol Laryngol 92:24-28

8. Daily WJR, Klaus M. Meyer HBP 1969 Apnea in premature infants: monitoring. incidence. heart rate changes, and an effect of environmental temperature. Pediatrics 43:510-518

9. Bertoittiere D. D'Allets A-M. Dehan M. Gaultier C 1990 Effects of increase in body temperature on the breathing pattern in premature infants. $J$ Dev Physiol 13:303-308

10. Perlstein PH, Edwards NK. Sutherland JM 1970 Apnea in premature infants and incubator air temperature. N Engl J Med 282:461-466

11. Sakaguchi S. Glotzbach SF. Heller HC 1987 Influence of hypothalamic and ambient temperatures on sleep in kangaroo rats. Am J Physiol 237:R80R88

12. Sichieri R. Schmidek WR 1984 Influence of ambient temperature on the sleepwakefulness cycle in the golden hamster. Physiol Behav 33:871-877

13. Szymusiak R, Satinoff E 1981 Maximal REM sleep time defines a narrower thermoneutral zone than does minimal metabolic rate. Physiol Behav 26:687-690

14. Scott SC, Inman DG, Moss IR 1990 Ontogeny of sleep/wake and cardiorespiratory behaviour in unanesthetized piglets. Respir Physiol 80:83-102

15. Barrand MA, Dauncey MJ, Ingram DL 1981 Changes in plasma noradrenaline and adrenaline associated with central and peripheral thermal stimuli in the pig. J Physiol 316:139-152

16. Lister G. Hoffman JIE. Rudolph AM 1974 Oxygen uptake in infants and children: a simple method for measurement. Pediatrics 53:656-662

17. Consolazio CF. Johnson RE. Pecora LJ 1963 Physiological Measurements of Metabolic Functions in Man. McGraw-Hill. New York

18. Kales A. Malmstrom EJ, Kee HK, Kales JD. Tan TL 1969 Effects of hypnotics on sleep patterns, dreaming, and mood state: laboratory and home studies. Biol Psychiat 1:235-241

19. Lees M. Olsen G, McGilliard K, Newcomb J, Sunderland C 1982 Chloral 
hydrate and the carbon dioxide chemoreceptor response: a study of puppies and infants. Pediatrics 70:447-449

20. Hunt C. Hazinski T. Gora P 1982 Experimental effects of chloral hydrate on ventilatory response to hypoxia and hypercarbia. Pediatr Res 16:79-8

21. Kuipers M, Whatson TS 1979 Sleep in piglets: an observational study. Appl Anim Ethol 5:145-151

22. Muzet A, Ehrhart J, Candas V, Libert JP. Vogt JJ 1983 REM sleep and ambient temperature in man. Int J Neurosci 18:117-126

23. Henane R, Buguet A, Roussel B, Bittel J 1977 Variations in evaporation and body temperatures during sleep in man. J Appl Physiol 42:50-55

24. Haskell EH, Palca JW, Walker JM. Berger RJ, Heller HC 1981 The effects of high and low ambient temperatures on human sleep stages. Electroencephalogr Clin Neurophysiol 51:494-501

25. Parmeggiani PL, Agnati LF. Zamboni G, Cianci T 1975 Hypothalamic temperature during the sleep cycle at different ambient temperatures. Electroencephalogr Clin Neurophysiol 38:589-596

26. Hammel HT, Jackson DC, Stolwijk JAJ, Hardy JD, Stromme SB 1963 Temperature regulation by hypothalamic proportional control with an adjustable set point. J Appl Physiol 18:1146-1154

27. Libert JP. Di Nisi J, Fukuda H. Muzet A. Ehrhart J, Amoros C 1988 Effect of continuous exposure on sleep stages in humans. Sleep 11:195-209

28. Ferguson J, Dement $W 1968$ Changes in the intensity of REM sleep with deprivation. Psychophysiology 4:380-38

29. Parmeggiani P, Rabini C 1970 Sleep and environmental temperature. Arch Ital Biol 108:369-387

30. Szymusiak R, Satinoff E, Schallert T, Whishaw IQ 1980 Brief skin temperature changes towards thermoneutrality trigger REM sleep in rats. Physiol Behav $25: 305-311$
31. Bruck K 1978 Heat Production and Temperature Regulation. Plenum Medical Book Company. New York, pp 455-498

32. Mount LE 1962 Evaporative heat loss in the new-born pig. J Physiol 164:274281

33. Montagna W, Yun JS 1964 The skin of the domestic pig. J Invest Dermatol 42:11-22

34. Barltrop D 1954 The relation between body temperature and respiration. J Physiol 125:12P-20P

35. Landis EM Long WL Dunn JW Jackson CL Meyer U 1926 Studies on the effects of baths on man. III. Effects of hot baths on respiration. blood and urine. Am J Physiol 76:35-48

36. Canet E, Gaultier C, D'Allest A-M, Dehan M 1989 Effects of sleep deprivation on respiratory events during sleep in healthy infants. J Appl Physiol 66:11581163

37. Phillipson EA, Sullivan CE, Read DJ, Murphy E, Kozar LF 1978 Ventilatory and waking responses to hypoxia in sleeping dogs. J Appl Physiol 44:512520

38. Fewall JE, Baker SE 1987 Arousal from sleep during rapidly developing hypoxemia in lambs. Pediatr Res 22:471-477

39. Jeffery HE, Read DJC 1980 Ventilatory responses of newborn calves to progressive hypoxia in quiet and active sleep. J Appl Physiol 48:892-895

40. Berthon-Jones M, Sullivan CE 1982 Ventilatory and arousal responses to hypoxia in sleeping humans. Am Rev Respir Dis 125:632-639

41. Barrington KJ, Allen RG 1992 Comparison of arousal responses to tracheal and face mask occlusions in sleeping newborn piglets. J Appl Physiol 72:2482-2486

42. Kelly DH. Golub H. Carley D. Shannon DC 1986 Pneumograms in infants who subsequently died of sudden infant death syndrome. J Pediatr 109 : $249-254$ 